DRAFT VERSION MARCH 27, 2022

Preprint typeset using LTEX style emulateapj v. 5/2/11

\title{
INTERPRETATION OF THE STRUCTURE FUNCTION OF ROTATION MEASURE IN THE INTERSTELLAR MEDIUM
}

\author{
SIYAO XU ${ }^{1}$ AND BING ZHANG ${ }^{1,2,3}$ \\ Draft version March 27, 2022
}

\begin{abstract}
The observed structure function (SF) of rotation measure (RM) varies as a broken power-law function of angular scales. The systematic shallowness of its spectral slope is inconsistent with the standard Kolmogorov scaling. This motivates us to examine the statistical analysis on RM fluctuations. The correlations of RM constructed by Lazarian \& Pogosyan (2016) are demonstrated to be adequate in explaining the observed features of RM SFs through a direct comparison between the theoretically obtained and observationally measured SF results. By segregating the density and magnetic field fluctuations and adopting arbitrary indices for their respective power spectra, we find that when the SFs of RM and emission measure have a similar form over the same range of angular scales, the statistics of the RM fluctuations reflect the properties of density fluctuations. RM SFs can be used to evaluate the mean magnetic field along the line of sight, but cannot serve as an informative source on the properties of turbulent magnetic field in the interstellar medium. We identify the spectral break of RM SFs as the inner scale of a shallow spectrum of electron density fluctuations, which characterizes the typical size of discrete electron density structures in the observed region.
\end{abstract}

Subject headings: ISM: magnetic fields - turbulence - methods: statistical

\section{INTRODUCTION}

Astrophysical turbulence, in spite of the stochastic nature, allows for a statistical study that can have access to its underlying regularities (Biskamp 2003; Brandenburg \& Lazarian 2013; Beresnyak \& Lazarian 2015). The turbulent spectrum, as a statistical measure of turbulence, contains a great wealth of information on the injection, nonlinear transfer, and dissipation of turbulent energy, and thus can characterize the essential properties of interstellar turbulence. The statistics of the turbulent velocity provide a direct diagnostic of the turbulent spectrum, but it is challenging to disentangle velocity and density contributions when utilizing spectroscopic data to obtain velocity statistics (Vestuto et al. 2003). Among the attempts to overcome this difficulty, new techniques, e.g., the Velocity Channel Analysis and Velocity Coordinate Spectrum, have been developed on a solid theoretical ground and successfully tested by numerical simulations (see a review by Lazarian 2009). On the other hand, the statistical study of density is rather straightforward and has attracted more attention (Goodman \& Narayan 1985; Spangler \& Gwinn 1990; Armstrong et al. 1995; Chepurnov \& Lazarian 2010). A Kolmogorov spectrum of the fluctuations in the interstellar plasma density is suggested by observational evidence. However, density is a passive scalar and the measure of density fluctuations can only be regarded as an indirect approach of tracing turbulence. Numerical studies show that the density spectrum significantly deviates from the velocity spectrum in supersonic turbulence (Cho \& Lazarian 2003; Beresnyak et al. 2005; Kowal et al. 2007).

Turbulence induces fluctuating magnetic field by the smallscale dynamo, and brings the magnetic energy up to the injection scale of turbulence through the inverse cascade (Cho et al. 2009; Beresnyak 2012; Zrake 2014). The gen-

\footnotetext{
${ }^{1}$ Department of Astronomy, School of Physics, Peking University, Beijing 100871, China; syxu@pku.edu.cn

${ }^{2}$ Kavli Institute for Astronomy and Astrophysics, Peking University, Beijing 100871, China

${ }^{3}$ Department of Physics and Astronomy, University of Nevada Las Vegas, NV 89154, USA; zhang@physics.unlv.edu
}

erated magnetic field in turn affects the properties of turbulence and converts the hydrodynamic turbulence into magnetohydrodynamic (MHD) turbulence, which is a common state of interstellar plasma. Achieving the spectral profile of turbulent magnetic field is crucial for studying the processes such as cosmic-ray scattering (Kóta \& Jokipii 2000), star formation (McKee 1999), and magnetic reconnection (Lazarian \& Vishniac 1999). It requires an adequate understanding of MHD turbulence. The point of contention is whether the scaling law for hydrodynamic turbulence is still valid in the context of MHD turbulence. Within theory's reach, Goldreich \& Sridhar (1995) pointed out that the transverse mixing motions of magnetic field lines in MHD turbulence preserve the character of hydrodynamic turbulent motions, thus the hydrodynamic turbulence scaling holds in the direction perpendicular to the local magnetic field. The Kolmogorov-type spectrum of density fluctuations in the magnetized interstellar plasma is in accordance with this theoretical expectation. Statistical analyses of the magnetic field data produced by numerical simulations support the theory (Cho \& Vishniac 2000; Cho et al. 2002; Maron \& Goldreich 2001). However, there is a shortage of observational evidence since magnetic field statistics are more poorly constrained from observations than velocity and density statistics. Magnetic field cannot be measured independently, but is intermixed with other quantities such as densities of relativistic electrons or thermal electrons. Only a theoretical model capable of reproducing the detected features of related observables can give us confidence in eliminating the inherent ambiguities and unveiling the physics in the measurements of turbulent magnetic fields.

Based on the modern understanding of MHD turbulence, Lazarian \& Pogosyan (2012, 2016) carried out comprehensive statistical studies on fluctuations in synchrotron intensity, synchrotron polarization, and Faraday measure. They provided a thorough exposition on the quantitative correlations between the statistics of synchrotron emission and characteristics of the underlying magnetic turbulence. Their synchrotron studies of turbulence and cosmic magnetic fields 
open the avenue to a wide range of astrophysical applications. In particular, Lazarian \& Pogosyan (2016) (hereafter LP16) presented the structure function (SF) analysis of rotation measures (RMs), including both cases with spatiallycoincident and spatially-separated synchrotron emission and Faraday rotation regions. The latter case can be applied to probing the turbulent magnetic fields embedded in the diffuse ionized component of the interstellar medium (ISM), when the observed Faraday rotation only contains the contribution from the Galaxy.

In practice, the SFs of RMs have been attained from a number of independent observations covering different scales and areas in the Galaxy (e.g., Simonetti et al. 1984; Simonetti \& Cordes 1986; Clegg et al. 1992; Minter \& Spangler 1996; Haverkorn et al. 2003, 2004; Sun \& Han 2004; Haverkorn et al. 2008; Rov et al.|2008; Stil 2009; Oppermann et al. 2012; see also, Han et al. 2004; Han 2009). In combination with the SFs of emission measures (EMs), it provides a possibility for determining the properties of turbulent magnetic fields in the ISM. The observations reveal some common features in the form of RM SFs as a function of angular scales: (1) The SF has a much shallower slope than that expected from the standard Kolmogorov power law. (2) The SF follows a broken power spectrum changing from a relatively steeper slope to a shallower one at a scale on the order of 1 pc. (3) The slope of the SF varies from region to region and has a dependence on Galactic latitude. (4) The slope of the SF tends to flatten at large angular scales. (5) The SF of EMs shows a similar slope to that of RMs detected from the same region. There has not been a compelling interpretation for these features in earlier literature. As an empirical attempt, Minter \& Spangler (1996) suggested that the broken power-law spectrum may result from the transition from three-dimensional to twodimensional filamentary turbulent structure, but this imposed turbulent structure, which may not be a common occurrence, fails to account for other observational features.

As the major impediment of the problem, both density and magnetic field fluctuations are imprinted in the observed RM fluctuations. The relative importance between them determines whether the behavior of RM SFs can effectively diagnose the turbulent magnetic fields. In the studies of, e.g. Simonetti et al. (1984); Lazarian \& Pogosyan (2016), the product of electron density and magnetic field has been treated as a composite quantity. In Minter \& Spangler (1996), the two are separated, but both fluctuations are assumed to conform to the Kolmogorov spectrum. In the current work, in order to resolve the respective influence of density and magnetic field fluctuations and gain a clear insight into the properties of their associated turbulence, we separate the density and magnetic field components in the statistical analysis of RM fluctuations, but do not restrict the scalings of their respective power spectra. Our goal is not only to seek understanding of the observed features of RM SFs, but also to clarify its relation with the turbulent density field and magnetic field. In Section 2, we present a statistical analysis of RM and EM fluctuations, and provide the expressions of their SFs with separate contributions from electron density and magnetic field. In Section 3 , we compare the analytical result with the measured RM and EM SFs from observations. Conclusions and discussions are given in Section 4.

\section{STATISTICAL ANALYSIS OF RM AND EM FLUCTUATIONS}

As the fundamental radio propagation measurements, the measure of magnetization (RM) and electron densities (EM) provide unique information on the magnetized turbulence in the diffusive, ionized component of the ISM. In astronomically convenient units, they are defined as

$$
\mathrm{RM}\left(\mathrm{rad} \mathrm{m}^{-2}\right)=0.81 \int_{0}^{L} n_{e}\left(\mathrm{~cm}^{-3}\right) B_{z}(\mu \mathrm{G}) d z(\mathrm{pc}),
$$

and

$$
\operatorname{EM}\left(\mathrm{pc} \mathrm{cm}^{-6}\right)=\int_{0}^{L}\left[n_{e}\left(\mathrm{~cm}^{-3}\right)\right]^{2} d z(\mathrm{pc}),
$$

where $n_{e}$ is electron density, $B_{z}$ is the line-of-sight (LOS) component of magnetic field, and $L$ is the path length through the Faraday rotating medium.

\subsection{SFs of RM and EM fluctuations}

For our statistical analysis of RM and EM fluctuations, we follow the approach employed by LP16 that deals with RM per unit length along LOS, namely, RM density $0.81\left(n_{e} B_{z}\right)$, and EM density $\left(n_{e}^{2}\right)$. We first treat them as composite quantities. We consider them to be statistically homogeneous and isotropic. This ensures that these quantities are invariant with respect to the LOS orientation.

We assume that the RM (EM) density can be described as a sum of its ensemble-average mean and zero mean fluctuations,

$$
\phi(\boldsymbol{X}, z)=\phi_{0}+\delta \phi(\boldsymbol{X}, z),\langle\delta \phi(\boldsymbol{X}, z)\rangle=0,
$$

where $\boldsymbol{X}$ denotes the position on the plane of sky and $z$ is the distance along the LOS. We use $\langle\ldots$.$\rangle to denote an ensem-$ ble average. As the real-space statistical tool, the two-point correlation function $(\mathrm{CF})$ is

$$
\xi(\boldsymbol{R}, \Delta z)=\left\langle\phi\left(\boldsymbol{X}_{\mathbf{1}}, z_{1}\right) \phi\left(\boldsymbol{X}_{\mathbf{2}}, z_{2}\right)\right\rangle,
$$

for RM (EM) density, and

$$
\widetilde{\xi}(\boldsymbol{R}, \Delta z)=\left\langle\delta \phi\left(\boldsymbol{X}_{\mathbf{1}}, z_{1}\right) \delta \phi\left(\boldsymbol{X}_{\mathbf{2}}, z_{2}\right)\right\rangle
$$

for RM (EM) density fluctuations. The two are related as

$$
\widetilde{\xi}(\boldsymbol{R}, \Delta z)=\xi(\boldsymbol{R}, \Delta z)-\phi_{0}^{2},
$$

with $\boldsymbol{R}=\boldsymbol{X}_{\mathbf{1}}-\boldsymbol{X}_{\mathbf{2}}$ and $\Delta z=z_{1}-z_{2}$. The SF of RM (EM) density and RM (EM) density fluctuations are identical,

$$
d(\boldsymbol{R}, \Delta z)=\widetilde{d}(\boldsymbol{R}, \Delta z)=\left\langle\left[\delta \phi\left(\boldsymbol{X}_{\mathbf{1}}, z_{1}\right)-\delta \phi\left(\boldsymbol{X}_{\mathbf{2}}, z_{2}\right)\right]^{2}\right\rangle .
$$

According to the statistical descriptions presented in LP16, we adopt a power-law model of CF and SF, which is adequate for characterizing the scaling properties of turbulence. Their forms are

$$
\begin{aligned}
& \widetilde{\xi}_{\phi}(\boldsymbol{R}, \Delta z)=\sigma_{\phi}^{2} \frac{r_{\phi}^{m_{\phi}}}{r_{\phi}^{m_{\phi}}+\left(R^{2}+\Delta z^{2}\right)^{m_{\phi} / 2}}, \\
& \widetilde{d}_{\phi}(\boldsymbol{R}, \Delta z)=2 \sigma_{\phi}^{2} \frac{\left(R^{2}+\Delta z^{2}\right)^{m_{\phi} / 2}}{r_{\phi}^{m_{\phi}}+\left(R^{2}+\Delta z^{2}\right)^{m_{\phi} / 2}},
\end{aligned}
$$

with the variance of fluctuations defined as

$$
\sigma_{\phi}^{2}=0.81^{2}\left\langle\delta\left(n_{e} B_{z}\right)^{2}\right\rangle
$$

for RM density, and

$$
\sigma_{\phi}^{2}=\left\langle\delta\left(n_{e}^{2}\right)^{2}\right\rangle
$$


for EM density, where $r_{\phi}$ is the correlation scale of RM (EM) density fluctuations, and $m_{\phi}$ is the index of their power-law functions in real space. Under the condition of statistical homogeneity, we see from above expressions that $\mathrm{CF}$ and SF only depend on the relative separation distance instead of the separation vector between the two points.

The power spectrum in Fourier space $E(k) \sim k^{\alpha}$ is complementary to CF and SF. Since CF and SF respectively apply to small-scale and large-scale dominated statistics, a shallow $(\alpha>-3)$ spectrum is more properly described by $\mathrm{CF}$, while a steep $(\alpha<-3)$ spectrum more favors SF treatment. Only when the cutoffs of $\mathrm{CF}$ at small scales and $\mathrm{SF}$ at large scales are both defined, can CF and SF be related and employed simultaneously (see detailed discussions in Lazarian \& Pogosyan 2004, 2006). From Eq. (8), we find

$$
\begin{aligned}
& \widetilde{\xi}_{\phi}(0)=\sigma_{\phi}^{2}, \\
& \widetilde{d}_{\phi}(\infty)=2 \sigma_{\phi}^{2},
\end{aligned}
$$

and thus the $\mathrm{CF}$ and $\mathrm{SF}$ are related by

$$
\begin{aligned}
& \widetilde{d}_{\phi}(\boldsymbol{R}, \Delta z)=2\left[\widetilde{\xi}_{\phi}(0)-\widetilde{\xi}_{\phi}(\boldsymbol{R}, \Delta z)\right], \\
& \widetilde{\xi}_{\phi}(\boldsymbol{R}, \Delta z)=\frac{1}{2}\left[\widetilde{d}_{\phi}(\infty)-\widetilde{d}_{\phi}(\boldsymbol{R}, \Delta z)\right] .
\end{aligned}
$$

The relation between the CF (SF) index $m_{\phi}$ and the spectral index $\alpha$ depends on whether the turbulent spectrum is shallow or steep (Lazarian \& Pogosyan 2006),

$$
\alpha= \begin{cases}m_{\phi}-N, & \alpha>-3 \\ -m_{\phi}-N, & \alpha<-3\end{cases}
$$

where $N$ is the dimensionality of space. In the case of threedimensional Kolmogorov turbulence with $\alpha=-11 / 3$, the corresponding value of $m_{\phi}$ is $2 / 3$. Regarding the correlation scale $r_{\phi}$, as pointed out by LP16, it corresponds to the energy dissipation scale for a shallow spectrum at wavenumbers smaller than $1 / r_{\phi}$, and the injection scale of turbulent energy for a steep spectrum at wavenumbers larger than $1 / r_{\phi}$. Strictly speaking, the forms of CF and SF given in Eq. (8) are only applicable in the inertial range of the spectrum. For scales below the inner scale $r_{\phi}$ of a shallow spectrum and above the outer scale $r_{\phi}$ of a steep spectrum, the exact forms of $\mathrm{CF}$ and $\mathrm{SF}$ depend on the specific dissipation and injection processes of turbulent energy.

The total RM and EM are the integrals of RM and EM densities over the path length along the LOS and thus their SFs have a dependence on the integration path. In a simple case where there is only one single Faraday rotating screen along the LOS with a thickness $L$, as derived in LP16, the SF for the fluctuations of RM (EM) is given by

$D_{\Delta \Phi}(\boldsymbol{R}, L, L)=2 \int_{0}^{L} d \Delta z(L-\Delta z)\left[\widetilde{\xi}_{\phi}(0, \Delta z)-\widetilde{\xi}_{\phi}(\boldsymbol{R}, \Delta z)\right]$.

Notice that the SF for RM (EM) has the same form as above due to $\widetilde{\xi}_{\phi}(0, \Delta z)-\widetilde{\xi}_{\phi}(\boldsymbol{R}, \Delta z)=\xi_{\phi}(0, \Delta z)-\xi_{\phi}(\boldsymbol{R}, \Delta z)$. A more complicated expression of $D_{\Delta \Phi}$ applicable to the situation with the synchrotron radiation and Faraday rotation taking place in the same volume is available in LP16.

We consider a thick Faraday screen with $L>r_{\phi}$, which is common to extragalactic sources. After inserting Eq. (8a), the SF from Eq. 114 has asymptotic expressions in different ranges of $R$ (see Appendix C in LP16),

$$
D_{\Delta \Phi}(R)= \begin{cases}2 \sigma_{\phi}^{2} L R\left(\frac{R}{r_{\phi}}\right)^{m_{\phi}}, & R<r_{\phi}<L,(15 \mathrm{a}) \\ 2 \sigma_{\phi}^{2} L R\left(\frac{R}{r_{\phi}}\right)^{-m_{\phi}}, & r_{\phi}<R<L,(15 \mathrm{~b}) \\ 2 \sigma_{\phi}^{2} L^{2}\left(\frac{L}{r_{\phi}}\right)^{-m_{\phi}}, & r_{\phi}<L<R .(15 \mathrm{c})\end{cases}
$$

Regarding its dependence on $R$, the slope changes from $1+m_{\phi}$ to $1-m_{\phi}$ when $R$ reaches the correlation scale $r_{\phi}$, and flattens when $R$ exceeds $L$. It is necessary to point out that, as we discussed above, the expression of $D_{\Delta \Phi}(R)$ at $R<r_{\phi}$ in the case of a shallow spectrum of RM (EM) density fluctuations and those at $R>r_{\phi}$ in the case of a steep spectrum are not robust. In the following calculations we assume that the same power-law model of $\widetilde{\xi}_{\phi}(\boldsymbol{R}, \Delta z)$ can be extensively applied beyond the inertial range of turbulence, keeping in mind that $m_{\phi}$ has an adjustable value in different ranges of scales, and we will discuss the modifications in a more realistic situation in Section 3 .

For an observer sitting in the Galaxy, if the Faraday rotation effect for the extragalactic sources mainly arises from the Galaxy, the angular separation $\theta$ between a pair of LOSs through the ISM coincides with the ratio of the projected distance and geometrical depth of the Galactic Faraday material, i.e., $R / L$. Thus, Eq. (15) can be recast into

$$
D_{\Delta \Phi}(\theta)=\left\{\begin{aligned}
2 \sigma_{\phi}^{2} L^{2}\left(\frac{L}{r_{\phi}}\right)^{m_{\phi}} \theta^{1+m_{\phi}}, & \\
& \theta<\frac{r_{\phi}}{L}<1, \\
2 \sigma_{\phi}^{2} L^{2}\left(\frac{L}{r_{\phi}}\right)^{-m_{\phi}} \theta^{1-m_{\phi}}, & \\
& \frac{r_{\phi}}{L}<\theta<1, \\
2 \sigma_{\phi}^{2} L^{2}\left(\frac{L}{r_{\phi}}\right)^{-m_{\phi}}, & \\
& \theta>1>\frac{r_{\phi}}{L} .
\end{aligned}\right.
$$

If the underlying turbulence conforms to the Kolmogorov scaling, there is $m_{\phi}=2 / 3$, so we expect that the SF exhibits a broken slope changing from $5 / 3$ at $\theta<r_{\phi} / L$ to $1 / 3$ at $r_{\phi} / L<\theta<1$, and remains unchanged at $\theta>1$.

The above conversion from the linear scale $R$ to angular scale $\theta$ should be adjusted when the LOSs intersect a Faraday screen at a distance from the observer. The observed angular separation of a source pair becomes

$$
\theta=\frac{R}{L+L_{f}},
$$

where $L_{f}$ is the distance from the Faraday screen to the observer. By substituting

$$
L^{\prime}=L+L_{f}, \quad \zeta=\frac{L}{L^{\prime}},
$$

and inserting

$$
R=L^{\prime} \theta
$$


Eq. (15) is reformulated as

$$
D_{\Delta \Phi}(\theta)=\left\{\begin{aligned}
2 \sigma_{\phi}^{2} \zeta L^{\prime 2}\left(\frac{L^{\prime}}{r_{\phi}}\right)^{m_{\phi}} \theta^{1+m_{\phi}}, & \\
& \theta<\frac{r_{\phi}}{L^{\prime}}<\zeta,(20 \mathrm{a}) \\
2 \sigma_{\phi}^{2} \zeta L^{\prime 2}\left(\frac{L^{\prime}}{r_{\phi}}\right)^{-m_{\phi}} \theta^{1-m_{\phi}}, & \\
& \frac{r_{\phi}}{L^{\prime}}<\theta<\zeta, \text { (20b) } \\
2 \sigma_{\phi}^{2} L^{2}\left(\frac{L}{r_{\phi}}\right)^{-m_{\phi},}, & \theta>\zeta>\frac{r_{\phi}}{L^{\prime}} . \text { (20c) }
\end{aligned}\right.
$$

The actual value of $\zeta$ can be evaluated from observations as the angular scale beyond which $D_{\Delta \Phi}(\theta)$ has a zero slope. We notice that Eq. (16) corresponds to the specialization of the above equation at $\zeta=1$, applicable to both extragalactic sources with little internal Faraday rotation and Galactic sources. In the opposite limit, for an extended extragalactic source with high internal Faraday rotation (within which the multiple RM components are correlated) and relatively negligible Galactic contribution, $\zeta$ can be much less than unity, from which and the estimated size of the radiation emitting region $(\sim L)$, the location of the Faraday screen, i.e., the distance of the source, can potentially be obtained. But such an observation requires very high angular resolution due to the large distance of the extragalactic source.

Besides the case with a single thick Faraday screen, which is the focus of this paper, analyses for other realizations with, e.g., a thin Faraday screen $\left(L<r_{\phi}\right)$ or a single $\operatorname{LOS}(R=0)$ are also provided in LP16, which can be widely applied to different observational situations.

\subsection{SFs with separate contributions from electron density and magnetic field}

The above approach straightforwardly reveals the dependence of the slope and amplitude of the RM SF on the spectral characteristics of RM density fluctuations. But it has the disadvantage that from the observed RM SF, the relative significance between density and magnetic field fluctuations cannot be readily discerned. We next carry out an analogous derivation of SFs of RM and EM as above, but separate the contributions from density and magnetic field.

Similarly, we assume that the electron density and LOS component of magnetic field are described by

$$
n_{e}=n_{e 0}+\delta n_{e}, \quad B_{z}=B_{z 0}+\delta B_{z},
$$

and

$$
\left\langle\delta n_{e}\right\rangle=0,\left\langle\delta B_{z}\right\rangle=0,
$$

such that we have the product $n_{e} B_{z}$

$$
\begin{aligned}
n_{e} B_{z} & =\left(n_{e 0}+\delta n_{e}\right)\left(B_{z 0}+\delta B_{z}\right) \\
& =n_{e 0} B_{z 0}+n_{e 0} \delta B_{z}+B_{z 0} \delta n_{e}+\delta n_{e} \delta B_{z},
\end{aligned}
$$

and the squared $n_{e}$

$$
n_{e}^{2}=n_{e 0}^{2}+\delta n_{e}^{2}+2 n_{e 0} \delta n_{e} .
$$

It follows that the CFs of RM and EM densities become (see detailed calculations given in the Appendix)

$$
\xi_{\phi}(\mathrm{RM})=0.81^{2}\left(n_{e 0}^{2} B_{z 0}^{2}+n_{e 0}^{2} \widetilde{\xi}_{B}+B_{z 0}^{2} \widetilde{\xi}_{n}+\widetilde{\xi}_{n} \widetilde{\xi}_{B}\right),
$$

and

$$
\xi_{\phi}(\mathrm{EM})=\left(n_{e 0}^{2}+\left\langle\delta n_{e}^{2}\right\rangle\right)^{2}+2 \widetilde{\xi}_{n}^{2}+4 n_{e 0}^{2} \widetilde{\xi}_{n}
$$

Here $\widetilde{\xi}_{n}$ and $\widetilde{\xi}_{B}$ are CFs for fluctuations in $n_{e}$ and $B_{z}$. By adopting the same model of $\mathrm{CF}$ as introduced in Eq. (8a), we have

$$
\begin{aligned}
\widetilde{\xi}_{n}(\boldsymbol{R}, \Delta z) & =\left\langle\delta n_{e}\left(\boldsymbol{X}_{\mathbf{1}}, z_{1}\right) \delta n_{e}\left(\boldsymbol{X}_{\mathbf{2}}, z_{2}\right)\right\rangle \\
& =\sigma_{n}^{2} \frac{r_{n}^{m_{n}}}{r_{n}^{m_{n}}+\left(R^{2}+\Delta z^{2}\right)^{m_{n} / 2}}, \\
\widetilde{\xi}_{B}(\boldsymbol{R}, \Delta z) & =\left\langle\delta B_{z}\left(\boldsymbol{X}_{\mathbf{1}}, z_{1}\right) \delta B_{z}\left(\boldsymbol{X}_{\mathbf{2}}, z_{2}\right)\right\rangle \\
& =\sigma_{B}^{2} \frac{r_{B}^{m_{B}}}{r_{B}^{m_{B}}+\left(R^{2}+\Delta z^{2}\right)^{m_{B} / 2}},
\end{aligned}
$$

with their respective correlation lengths $r_{n}, r_{B}$, power-law indices $m_{n}, m_{B}$, and variances of fluctuations

$$
\sigma_{n}^{2}=\left\langle\delta n_{e}^{2}\right\rangle, \quad \sigma_{B}^{2}=\left\langle\delta B_{z}^{2}\right\rangle
$$

Under the condition of relatively small fluctuations, the linear terms in $\xi_{\phi}(\mathrm{RM})$ (Eq. (25)) and $\xi_{\phi}(\mathrm{EM})$ (Eq. (26)) play a dominant role in determining the resultant SFs of RM and EM. Combining Eq. (14) with Eq. (25) and (26), we obtain analytical estimates,

$$
\begin{aligned}
& D_{\mathrm{RM}} \approx \\
& 2 \times 0.81^{2} n_{e 0}^{2} \int_{0}^{L} d \Delta z(L-\Delta z)\left[\widetilde{\xi}_{B}(0, \Delta z)-\widetilde{\xi}_{B}(\boldsymbol{R}, \Delta z)\right] \\
& +2 \times 0.81^{2} B_{z 0}^{2} \int_{0}^{L} d \Delta z(L-\Delta z)\left[\widetilde{\xi}_{n}(0, \Delta z)-\widetilde{\xi}_{n}(\boldsymbol{R}, \Delta z)\right],
\end{aligned}
$$

and

$$
D_{\mathrm{EM}} \approx 2 \times 4 n_{e 0}^{2} \int_{0}^{L} d \Delta z(L-\Delta z)\left[\widetilde{\xi}_{n}(0, \Delta z)-\widetilde{\xi}_{n}(\boldsymbol{R}, \Delta z)\right] .
$$

By comparing Eq. (14), (16), and (29), the simplified expressions of $D_{\mathrm{RM}}$ in different asymptotic regimes can then be 
derived:

$$
D_{\mathrm{RM}}(\theta)=\left\{\begin{array}{c}
2 \times 0.81^{2} L^{2}\left[n_{e 0}^{2} \sigma_{B}^{2}\left(\frac{L}{r_{B}}\right)^{m_{B}} \theta^{1+m_{B}}\right. \\
\left.+B_{z 0}^{2} \sigma_{n}^{2}\left(\frac{L}{r_{n}}\right)^{m_{n}} \theta^{1+m_{n}}\right], \\
\theta<\frac{r_{n}}{L}<\frac{r_{B}}{L}<1, \text { (31a) } \\
2 \times 0.81^{2} L^{2}\left[n_{e 0}^{2} \sigma_{B}^{2}\left(\frac{L}{r_{B}}\right)^{m_{B}} \theta^{1+m_{B}}\right. \\
\left.+B_{z 0}^{2} \sigma_{n}^{2}\left(\frac{L}{r_{n}}\right)^{-m_{n}} \theta^{1-m_{n}}\right], \\
\frac{r_{n}}{L}<\theta<\frac{r_{B}}{L}<1,(31 \mathrm{~b}) \\
2 \times 0.81^{2} L^{2}\left[n_{e 0}^{2} \sigma_{B}^{2}\left(\frac{L}{r_{B}}\right)^{-m_{B}} \theta^{1-m_{B}}\right. \\
\left.+B_{z 0}^{2} \sigma_{n}^{2}\left(\frac{L}{r_{n}}\right)^{-m_{n}} \theta^{1-m_{n}}\right], \\
\frac{r_{B}}{L}<\theta<1, \quad(31 \mathrm{c}) \\
2 \times 0.81^{2} L^{2}\left[n_{e 0}^{2} \sigma_{B}^{2}\left(\frac{L}{r_{B}}\right)^{-m_{B}}\right. \\
\left.+B_{z 0}^{2} \sigma_{n}^{2}\left(\frac{L}{r_{n}}\right)^{-m_{n}}\right], \\
\theta>1>\frac{r_{B}}{L} .(31 \mathrm{~d})
\end{array}\right.
$$

In the above expression, we consider the condition $r_{n}<r_{B}$. The opposite case with $r_{B}<r_{n}$ can be similarly formulated:

$$
D_{\mathrm{RM}}(\theta)=\left\{\begin{array}{c}
2 \times 0.81^{2} L^{2}\left[n_{e 0}^{2} \sigma_{B}^{2}\left(\frac{L}{r_{B}}\right)^{m_{B}} \theta^{1+m_{B}}\right. \\
\left.+B_{z 0}^{2} \sigma_{n}^{2}\left(\frac{L}{r_{n}}\right)^{m_{n}} \theta^{1+m_{n}}\right], \\
\theta<\frac{r_{B}}{L}<\frac{r_{n}}{L}<1,(32 \mathrm{a}) \\
2 \times 0.81^{2} L^{2}\left[n_{e 0}^{2} \sigma_{B}^{2}\left(\frac{L}{r_{B}}\right)^{-m_{B}} \theta^{1-m_{B}}\right. \\
\left.+B_{z 0}^{2} \sigma_{n}^{2}\left(\frac{L}{r_{n}}\right)^{m_{n}} \theta^{1+m_{n}}\right], \\
\frac{r_{B}}{L}<\theta<\frac{r_{n}}{L}<1,(32 \mathrm{~b}) \\
2 \times 0.81^{2} L^{2}\left[n_{e 0}^{2} \sigma_{B}^{2}\left(\frac{L}{r_{B}}\right)^{-m_{B}} \theta^{1-m_{B}}\right. \\
\left.+B_{z 0}^{2} \sigma_{n}^{2}\left(\frac{L}{r_{n}}\right)^{-m_{n}} \theta^{1-m_{n}}\right], \\
\frac{r_{n}}{L}<\theta<1, \quad(32 \mathrm{c}) \\
2 \times 0.81^{2} L^{2}\left[n_{e 0}^{2} \sigma_{B}^{2}\left(\frac{L}{r_{B}}\right)^{-m_{B}}\right. \\
\left.+B_{z 0}^{2} \sigma_{n}^{2}\left(\frac{L}{r_{n}}\right)^{-m_{n}}\right], \\
\theta>1>\frac{r_{n}}{L} . \quad(32 \mathrm{~d})
\end{array}\right.
$$

It reveals that the total $D_{\mathrm{RM}}$ is the superposition of two components that are related to the fluctuations in electron density and the LOS component of magnetic field, respectively. In an exceptional situation with $r_{n}=r_{B}$ and $m_{n}=m_{B}$, namely, density and magnetic turbulence share the same spectral scaling law, the expression of $D_{\mathrm{RM}}(\theta)$ in Eq. (16) can be recovered from either Eq. (31) or Eq. (32), and accordingly the CF parameters of RM density fluctuations can be more explicitly written as

$$
\begin{aligned}
& \sigma_{\phi}^{2}=0.81^{2}\left(n_{e 0}^{2} \sigma_{B}^{2}+B_{z 0}^{2} \sigma_{n}^{2}\right), \\
& r_{\phi}=r_{n}=r_{B}, \\
& m_{\phi}=m_{n}=m_{B} .
\end{aligned}
$$

This is only valid under rather restrictive circumstances. More generally, one would expect that the spectra for fluctuating density and magnetic field are not aligned and the behavior of $D_{\mathrm{RM}}$ depends on the relative importance between $B_{z 0}^{2} \sigma_{n}^{2}$ and $n_{e 0}^{2} \sigma_{B}^{2}$. For example, when $B_{z 0}^{2} \sigma_{n}^{2}$ is significantly larger than $n_{e 0}^{2} \sigma_{B}^{2}$, that is, the relative density fluctuations are much stronger in comparison with relative magnetic field fluctuations,

$$
\frac{\sigma_{n}^{2}}{n_{e 0}^{2}} \gg \frac{\sigma_{B}^{2}}{B_{z 0}^{2}},
$$

density fluctuations dictate the behavior of $D_{\mathrm{RM}}$. Both Eq. (31) and (32) approximately go back to Eq. (16), and the CF parameters of RM density fluctuations are equivalent to

$$
\begin{aligned}
& \sigma_{\phi}^{2}=0.81^{2} B_{z 0}^{2} \sigma_{n}^{2}, \\
& r_{\phi}=r_{n}, \\
& m_{\phi}=m_{n} .
\end{aligned}
$$

In this situation, magnetic field fluctuations are basically not responsible for the observed RM SFs.

As regards the SF of EM, combining Eq. (14), (16), and (30) yields

$$
D_{\mathrm{EM}}(\theta)=\left\{\begin{array}{c}
8 n_{e 0}^{2} \sigma_{n}^{2} L^{2}\left(\frac{L}{r_{n}}\right)^{m_{n}} \theta^{1+m_{n}}, \\
\theta<\frac{r_{n}}{L}<1, \\
8 n_{e 0}^{2} \sigma_{n}^{2} L^{2}\left(\frac{L}{r_{n}}\right)^{-m_{n}} \theta^{1-m_{n}}, \\
\frac{r_{n}}{L}<\theta<1, \\
8 n_{e 0}^{2} \sigma_{n}^{2} L^{2}\left(\frac{L}{r_{n}}\right)^{-m_{n}}, \\
\theta>1>\frac{r_{n}}{L} .
\end{array}\right.
$$

The similarity between the form of the above $D_{\mathrm{EM}}(\theta)$ and Eq. (16) suggests that the EM density fluctuations inherit the turbulence properties from the density field and their CF parameters are related as

$$
\begin{aligned}
& \sigma_{\phi}^{2}=4 n_{e 0}^{2} \sigma_{n}^{2}, \\
& r_{\phi}=r_{n}, \\
& m_{\phi}=m_{n} .
\end{aligned}
$$

If the situation described in Eq. (34) is realized, we expect that $D_{\mathrm{RM}}(\theta)$ and $D_{\mathrm{EM}}(\theta)$ measured from the same region in the sky exhibit a similar behavior in terms of the spectral slope and correlation scale corresponding to the break in slope. Their ratio

$$
\frac{D_{\mathrm{RM}}(\theta)}{D_{\mathrm{EM}}(\theta)}=\left(\frac{0.81 B_{z 0}}{2 n_{e 0}}\right)^{2}
$$


is associated with the mean plasma properties and determined by the ratio between the mean values of $B_{z}$ and $n_{e}$.

\section{COMPARISON WITH OBSERVATIONS}

Observationally determined SFs of RMs allow a quantitative test of the above analysis. Minter \& Spangler (1996) studied both RM and EM measurements for 38 extragalactic sources. The sample of source components are selected to have insignificant intrasource variations in RM and depolarization effects for the purpose of studying the Faraday rotation of the Galactic medium only. Another strong argument that the observed RM is dominated by the Galactic Faraday rotation is the evident dependence on the angular separation of the RM SF. The measured RM SF $D_{\mathrm{RM}}$ increases as a power-lawlike function of angular scale and is characterized by a break in its spectrum around $0.1^{\circ}$. The least-squares fit to the data above $0.1^{\circ}$ gives

$$
D_{\mathrm{RM}}\left(\theta>0.1^{\circ}\right)=(340 \pm 30)\left[\theta\left(^{\circ}\right)\right]^{0.64 \pm 0.06} \operatorname{rad}^{2} \mathrm{~m}^{-4} \text {. }
$$

The three data points below $0.1^{\circ}$ are insufficient for achieving a reliable fitting, but they clearly indicate a more steepened spectrum.

These observed features of RM SFs appear to be consistent with the theoretical expectations presented in Section 2. To enable a quantitative comparison, we employ the parameters provided in Minter \& Spangler (1996). Since the observed Faraday rotation for their sample is dominated by the magnetized ISM of our Galaxy, we adopt the average path length $2900 \mathrm{pc}$ through the ISM in the observed region as the depth $L$ of the Faraday screen. Besides, the very few measurements at small angular separations do not allow an accurate determination of the break point in the SF spectrum. We then choose the "outer scale" of turbulence of $3.6 \mathrm{pc}$ suggested in Minter \& Spangler (1996) as an experimental correlation scale $r_{\phi}$. The transition angle at the break is thus

$$
\theta_{\mathrm{tr}}=\frac{r_{\phi}}{L}=0.0711^{\circ}
$$

Given the coincidence $\theta=R / L$ for this observation, and the determination of $L$ and $r_{\phi}$, we can compare Eq. (39) with the functional form of $D_{\mathrm{RM}}$ at $\theta>\theta_{\text {tr }}$ from Eq. (16b), which directly yields the CF index and variance of RM density fluctuations,

$$
m_{\phi}=1-0.64=0.36, \quad \sigma_{\phi}=5.5 \times 10^{-2} \mathrm{rad} \mathrm{m}^{-2} \mathrm{pc}^{-1} .
$$

Evidently, the measured $m_{\phi}$ does not coincide with the prediction by Kolmogorov turbulence, which entails $m_{\phi}=2 / 3$ instead (see Section 2.1). In fact, since the range $R>r_{\phi}$ (i.e., $\theta>\theta_{\text {tr }}$ ) corresponds to the inertial range of a shallow spectrum of RM density fluctuations, the resulting spectral index is (Eq. 113a)

$$
\alpha=m_{\phi}-3=0.36-3=-2.64 .
$$

Meanwhile, the identification of the inertial range over the scales larger than $r_{\phi}$ indicates that $r_{\phi}$ is actually the inner scale rather than the outer scale of turbulence. Below $r_{\phi}$, the damping effect efficiently suppresses the fluctuations and steepens the spectral tail in the dissipation range. If we assume that the same scaling as in the inertial range can still be used at scales below but in the vicinity of $r_{\phi}$, inserting the values in Eq. (41) into the expression of $D_{\mathrm{RM}}$ at $\theta<\theta_{\mathrm{tr}}$ from Eq. (16a) leads to

$$
D_{\mathrm{RM}}\left(\theta<\theta_{\text {tr }}\right)=2.28 \times 10^{3}\left[\theta\left(^{\circ}\right)\right]^{1.36} \mathrm{rad}^{2} \mathrm{~m}^{-4} .
$$

Fig. 11plots $D_{\text {RM }}$ from Eq. (43) at $\theta<\theta_{\text {tr }}$ and Eq. (39) at $\theta>\theta_{\text {tr }}$, superposed with the observational data points taken from figure 5 in Minter \& Spangler (1996). It seems that both the amplitude and spectral slope of $D_{\mathrm{RM}}$ given by Eq. (43) are in good agreement with the observational result. However, due to the limited number of close source pairs, it is difficult to impose a strong constraint on the exact spectral slope in the dissipation range of turbulence. Nevertheless, the comparison illustrates that the theoretical model originally constructed by LP16 can satisfactorily interpret the observed RM SFs.

We exclude the possibility that $r_{\phi}$ is the outer scale of a steep spectrum, as in this case one would expect that the SF saturates at a constant value and flattens at $\theta>\theta_{\mathrm{tr}}$. Otherwise, the observed SF spectrum over an extended range of angular scales beyond $\theta_{\text {tr }}$ severely challenges the model for the energy injection of turbulence.

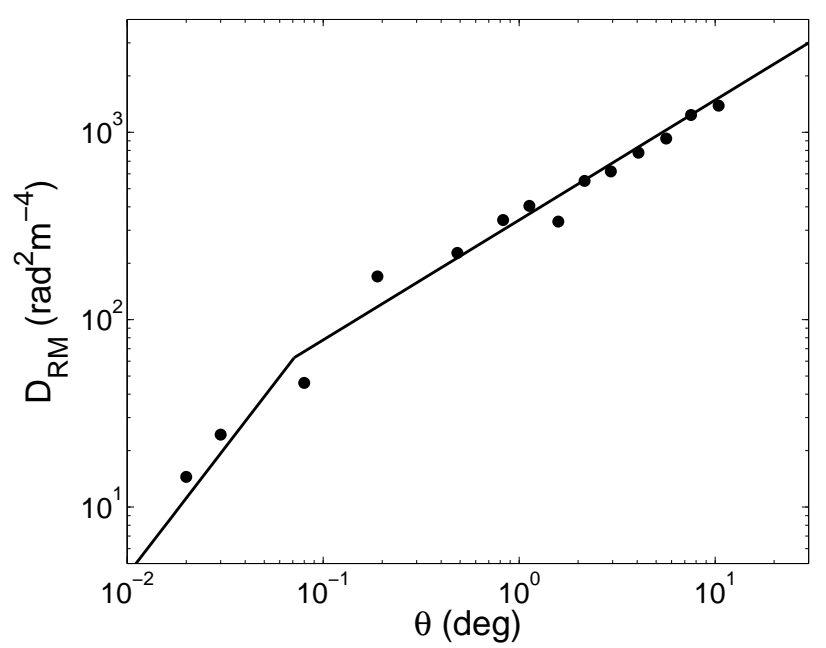

FIG. 1.- SFs of RMs vs. angular separation. The data points are taken from figure 5 in Minter \& Spangler (1996). The solid line corresponds to the theoretical formalisms Eq. (16a) and (16b).

From RM SFs alone, we are unable to identify the separate contributions due to density and magnetic field fluctuations. Hence, it is necessary to invoke SFs of EMs for an exclusive extraction of the electron density fluctuations. We now turn to the observationally measured EM SFs. A power-law representation with a similar slope to that of RM SFs fits the observations (Minter \& Spangler 1996),

$$
D_{\mathrm{EM}}(\theta)=(5.5 \pm 0.6)\left[\theta\left(^{\circ}\right)\right]^{0.73 \pm 0.08} \mathrm{~cm}^{-12} \mathrm{pc}^{2} .
$$

Due to the lower angular resolution of the EM data, the measurements of $D_{\mathrm{EM}}$ at small angular scales are absent, which prevents the detection of the possible break in its spectrum and determination of the correlation scale of EM density fluctuations. But the fitting by Eq. (44) is informative on the CF index of EM density fluctuations, which is also approximately the CF index of density fluctuations (see Eq. (37)). Comparing Eq. (16b) and (44) gives the value

$$
m_{\phi}=1-0.73( \pm 0.08)=0.27( \pm 0.08),
$$

close to that of RM density fluctuations (Eq. (41)). The similarity between the slopes of RM and EM SFs strongly suggests that the density fluctuations take a major part in composing the SF of RM. Presumably, the CF index and correlation 
scale obtained from RM SFs also match those quantities of density fluctuations (see Eq. (35)),

$$
m_{n}=0.36, \quad r_{n}=3.6 \mathrm{pc},
$$

and the EM SFs at $\theta>\theta_{\text {tr }}$ satisfy

$$
D_{\mathrm{EM}}(\theta)=5.5\left[\theta\left(^{\circ}\right)\right]^{0.64} \mathrm{~cm}^{-12} \mathrm{pc}^{2} .
$$

By comparing the above equation with Eq. (36b) and using $L=2900$ pc and values in Eq. (46), we find

$$
n_{e 0} \sigma_{n}=3.47 \times 10^{9} \mathrm{~m}^{-6} .
$$

Furthermore, under the condition of Eq. (34), we can safely neglect the terms associated with magnetic fluctuations in Eq. (31) and (32) due to their minor contribution. From Eq. (35) and (41), we get

$$
0.81 B_{z 0} \sigma_{n}=\sigma_{\phi}=5.5 \times 10^{-2} \mathrm{rad} \mathrm{m}^{-2} \mathrm{pc}^{-1},
$$

indicative of the fact that the level of RM density fluctuations is determined by the joint strength of fluctuating electron density and mean magnetic field along the LOS. Given the estimate of mean electron density $n_{e 0}, \sigma_{n}$ and $B_{z 0}$ can be both derived from the above two equations. However, the properties of the fluctuating component of magnetic field are poorly constrained by SFs of RMs.

The above results suggest that the observed RM SFs originate from the underlying shallow spectrum of density fluctuations, with the break in the slope of the SF corresponding to the inner scale of the density spectrum. Accordingly, the Faraday rotating medium that gives rise to the characteristics of RM SFs has an excess of dense structures at small scales comparable to $r_{\phi}$.

As a caveat to the applicability of the theoretical model, the uncertainties in the amplitude and correlation scale of turbulence obtained from observations are introduced by the choice of Faraday rotation depth $L$. The RM fluctuations traced by Galactic sources provide information about the small-scale turbulence within a specific region in the Galaxy. The Faraday rotating medium extends from the observer to sources and thus the sources' distances can be used as the estimate of $L$. Observations of extragalactic sources with marginal intrinsic Faraday rotation as in Minter \& Spangler (1996) can bring forth large-scale features of ISM turbulence. The depth of the Faraday screen is the path length throughout the Galaxy, and the sources' distances are irrelevant. But in the case with dominant intrinsic Faraday rotation, provided that the multiple RM components within one source can be resolved, the properties of turbulence in the source region are probed. Apart from $L$, the source's distance is also involved in the analysis (see Eq. (20).

\section{CONCLUSIONS AND DISCUSSION}

Following the model SF of RM fluctuations put forth by LP16, we proceed to carry out the SF analysis of RM and EM by separating the contributions from fluctuations of electron density and the LOS component of magnetic field, and we assess their relative importance in determining the form of SFs of RMs as a function of angular scales. We found that the SF of RM can be considered as a sum of two SFs stemming from the fluctuations of electron density and magnetic field, respectively. The turbulent spectrum of the density fluctuations can be extracted from the SF measurement of EM provided that the angular resolution is sufficient. Applying the analysis to observationally determined SFs of RMs shows that the model SF can consistently interpret the shape of SFs on different ranges of angular scales. Similar behavior of RM and EM SFs suggests that the observed SF of RM is mainly guided by fluctuating electron density and thus incapable of probing the nature of turbulent magnetic field.

As an observational fact, the changing shape of SFs of RMs with angular scales was earlier interpreted as arising from a transition from three-dimensional to two-dimensional turbulence (Minter \& Spangler 1996), or two spatially separate Faraday screens (Haverkorn et al. 2004). Without the intervention of two turbulent structures or two Faraday rotation regions, the power-law CF for RM density fluctuations introduced in LP16 can naturally reproduce the observed features and explain the broken power-law shape of the RM SF.

In earlier works, magnetic field is assumed to be frozen in matter, and thus both density and magnetic field fluctuations adhere to an identical power-law spectrum with the same spectral index and inner and outer scales. But this conjecture has been definitely rejected by observations on RM fluctuations. The Kolmogorov spectrum appears to be too steep to account for the shallow slope especially at scales larger than the spectral break, and it alone cannot serve as a satisfactory turbulent model to fit the various slopes of SFs from different observations. In fact, it has been known that the conventional flux-freezing concept breaks down in realistic MHD turbulence as the diffusion of magnetic field lines is mediated by fast magnetic reconnection, which is an intrinsic process inherent in MHD turbulence (Lazarian 2005, 2011; Lazarian et al. 2012; Eyink et al. 2013). It is more plausible that density and magnetic field fluctuations conform to distinct power spectra of turbulence. The dominant one between them is more important in determining the shape of the resultant SF spectrum of RM.

The similarity between the behavior of RM and EM SFs revealed by observations (e.g. Minter \& Spangler 1996; Haverkorn et al. 2004) indicates that the major contribution to the measured RM SFs comes from electron density fluctuations, which tend to follow a shallow spectrum of turbulence down to the dissipation scale corresponding to the spectral break of the RM SF. Both theoretical considerations and numerical simulations suggest that a shallow spectrum of density field can arise in compressible turbulent flows. Compressibility leads to the formation of clumpy density structures, with condensations embedded in relatively diffuse regions (Beresnyak et al. 2005; Kritsuk et al. 2007; Kowal et al. 2007; Falceta-Goncalves et al. 2014). The coupling between this density structure and local turbulent motions results in a steeper velocity power spectrum with a slope of $\sim-2$, but a much shallower power spectrum of the density field than the Kolmogorov $-5 / 3$ scaling for one-dimensional spectra. Moreover, the shallowness of the spectral slope of density fluctuations is strongly affected by magnetic field strength in subsonic turbulence and by sonic Mach number $M_{s}$ in supersonic turbulence (Kowal et al. 2007). For example, Kowal et al. (2007) observed a slope of $\sim-0.5$ for the onedimensional density spectrum obtained from the simulated supersonic turbulence with $M_{s}=7$, which is even shallower than the spectral slope indicated from the RM SFs measured by Minter \& Spangler (1996) (see Eq. (42)). The ISM is highly inhomogeneous with dense structures accumulated by shocks on small scales. Depending on the local compressibility, the density fluctuations and the resultant RM SFs can have spatially diverse spectral slopes. 
In contrast to density, magnetic field is better coupled with turbulent velocity field and regulates the turbulence properties, e.g., scale-dependent turbulent anisotropy, as the turbulent energy cascades down from large to small scales. The "Big Power Law in the Sky" extending from $10^{17} \mathrm{~m}$ to $10^{6} \mathrm{~m}$ (Armstrong et al. 1995; Chepurnov \& Lazarian 2010) suggests that the interstellar turbulence has a Galactic-scale ( $>100 \mathrm{pc}$ ) energy injection source and cascades toward very small scales. At the parsec scale of this global turbulent cascade, magnetic field fluctuations can have a lower amplitude compared with the enhanced amplitude of density fluctuations. These excessive density fluctuations are thus manifest by dominating the composite RM density fluctuations. The candidate regions corresponding to the localized enhanced turbulence in density field can be the extended envelopes of $\mathrm{H}$ II regions and the warm ionized medium of the McKee \& Ostriker (1977) model in the Galactic plane (Spangler 1991; Armstrong et al. 1995).

Chepurnov \& Lazarian (2010) carried out a remarkable extension of the Big Power Law in the Sky in Armstrong et al. (1995) up to $10^{17} \mathrm{~m}$ by using the data of the Wisconsin $\mathrm{H} \alpha$ Mapper (WHAM) and demonstrate a universal spectrum of interstellar density fluctuations consistent with Kolmogorov turbulence. On the contrary, observations of RM fluctuations at low Galactic latitudes (e.g. Simonetti \& Cordes 1986; Clegg et al. 1992; Haverkorn et al. 2004, 2008) imply a shallower spectrum than the Kolmogorov one. As a possible understanding of this contradiction, for LOSs that traverse the supersonic turbulent flows through the Galactic plane, the RM results are mainly governed by density fluctuations, which we do not expect to be compatible with the Kolmogorov spectrum as they are more independent from turbulent velocity and sensitive to the local compressibility of the medium. Chepurnov \& Lazarian (2010) analyzed the WHAM data at high Galactic latitudes and avoided the contamination from the $\mathrm{H}$ II regions in the Galactic plane. As a result, they achieved one universal turbulent spectrum throughout the Galaxy. Their result is also in agreement with the study of velocity turbulence by using the velocity coordinate spectrum technique in Chepurnov et al. (2010). So great caution is needed when one uses RM fluctuations to probe the turbulent magnetic fields in the Galaxy, not only because the fluctuating density instead of fluctuating magnetic field can dominate the observed RM SF behavior, but also because more complexity can be introduced by additional structures of turbulence embedded in the observed region. They both hinder the recovery of the underlying spectrum of magnetized turbulence.

We identify the transition scale on the order of $1 \mathrm{pc}$ at the spectral break of RM SFs as the inner scale of a shallow electron density spectrum over larger scales, instead of the injection scale of RM fluctuations at smaller scales as determined by Haverkorn et al. (2004, 2008). In a partially ionized ISM, the cascade of MHD turbulence is severely damped due to ion-neutral collisions. Below the scale where neutral fluid decouples from ion-electron fluid, the MHD turbulence in ion-electron fluid is efficiently suppressed by ion-neutral collisional damping, but the cascade of hydrodynamic turbulence in neutrals proceeds down to the viscous cutoff. The dissipation scale inferred from RM SFs we obtained in this work is consistent with the ion-neutral collisional damping scale of MHD turbulence in the warm neutral phase of the ISM calculated by $\mathrm{Xu}$ et al. (2015). Plausibly, this consistency could suggest that the inner scale to electron density fluctuations of $\sim 1$ pc characterizes the typical and also minimum scale of discrete structures of excess electron densities in the observed region, while smaller condensations mostly consist of neutrals and are driven by gravitational contraction instead of supersonic motions.

The authors thank the anonymous referee for the valuable comments. This work is partially supported by the National Basic Research Program (973 Program) of China under grant No. 2014CB845800.

\section{APPENDIX}

\section{CALCULATIONS FOR THE CFS OF RM AND EM DENSITIES}

The CFs of RM and EM densities are calculated as

$$
\begin{aligned}
& \xi_{\phi}(\mathrm{RM}) \\
= & 0.81^{2}\left\langle\left(n_{e} B_{z}\right)\left(\boldsymbol{X}_{\mathbf{1}}, z_{1}\right)\left(n_{e} B_{z}\right)\left(\boldsymbol{X}_{\mathbf{2}}, z_{2}\right)\right\rangle \\
= & 0.81^{2}\left[n_{e 0}^{2} B_{z 0}^{2}+n_{e 0}^{2}\left\langle\delta B_{z}\left(\boldsymbol{X}_{\mathbf{1}}, z_{1}\right) \delta B_{z}\left(\boldsymbol{X}_{\mathbf{2}}, z_{2}\right)\right\rangle+B_{z 0}^{2}\left\langle\delta n_{e}\left(\boldsymbol{X}_{\mathbf{1}}, z_{1}\right) \delta n_{e}\left(\boldsymbol{X}_{\mathbf{2}}, z_{2}\right)\right\rangle\right. \\
& \left.+\left\langle\delta n_{e}\left(\boldsymbol{X}_{\mathbf{1}}, z_{1}\right) \delta B_{z}\left(\boldsymbol{X}_{\mathbf{1}}, z_{1}\right) \delta n_{e}\left(\boldsymbol{X}_{\mathbf{2}}, z_{2}\right) \delta B_{z}\left(\boldsymbol{X}_{\mathbf{2}}, z_{2}\right)\right\rangle\right] \\
= & 0.81^{2}\left(n_{e 0}^{2} B_{z 0}^{2}+n_{e 0}^{2} \widetilde{\xi}_{B}+B_{z 0}^{2} \widetilde{\xi}_{n}+\widetilde{\xi}_{n B}\right) \\
= & 0.81^{2}\left(n_{e 0}^{2} B_{z 0}^{2}+n_{e 0}^{2} \widetilde{\xi}_{B}+B_{z 0}^{2} \widetilde{\xi}_{n}+\widetilde{\xi}_{n} \widetilde{\xi}_{B}\right),
\end{aligned}
$$

and

$$
\begin{aligned}
& \xi_{\phi}(\mathrm{EM}) \\
= & \left\langle\left(n_{e}^{2}\right)\left(\boldsymbol{X}_{\mathbf{1}}, z_{1}\right)\left(n_{e}^{2}\right)\left(\boldsymbol{X}_{\mathbf{2}}, z_{2}\right)\right\rangle \\
= & n_{e 0}^{4}+2 n_{e 0}^{2}\left\langle\delta n_{e}^{2}\right\rangle+\left\langle\delta n_{e}\left(\boldsymbol{X}_{\mathbf{1}}, z_{1}\right)^{2} \delta n_{e}\left(\boldsymbol{X}_{\mathbf{2}}, z_{2}\right)^{2}\right\rangle+4 n_{e 0}^{2}\left\langle\delta n_{e}\left(\boldsymbol{X}_{\mathbf{1}}, z_{1}\right) \delta n_{e}\left(\boldsymbol{X}_{\mathbf{2}}, z_{2}\right)\right\rangle \\
= & n_{e 0}^{4}+2 n_{e 0}^{2}\left\langle\delta n_{e}^{2}\right\rangle+\widetilde{\xi}_{n n}+4 n_{e 0}^{2} \widetilde{\xi}_{n} \\
= & \left(n_{e 0}^{2}+\left\langle\delta n_{e}^{2}\right\rangle\right)^{2}+2 \widetilde{\xi}_{n}^{2}+4 n_{e 0}^{2} \widetilde{\xi}_{n} .
\end{aligned}
$$

In our derivations above, the three-point correlations are neglected under the assumption that the turbulent fluctuations are Gaussian processes, and the turbulent density and magnetic field are assumed to be uncorrelated (Minter \& Spangler 1996). Following 
the four-point correlations as employed in Minter \& Spangler (1996), the expressions of $\widetilde{\xi}_{n B}$ and $\widetilde{\xi}_{n n}$ in the above equations are

$$
\widetilde{\xi}_{n B}=\widetilde{\xi}_{n} \widetilde{\xi}_{B}, \quad \widetilde{\xi}_{n n}=\left\langle\delta n_{e}^{2}\right\rangle^{2}+2 \widetilde{\xi}_{n}^{2} .
$$

\section{REFERENCES}

Armstrong, J. W., Rickett, B. J., \& Spangler, S. R. 1995, ApJ, 443, 209 Beresnyak, A. 2012, Physical Review Letters, 108, 035002

Beresnyak, A., \& Lazarian, A. 2015, in Astrophysics and Space Science Library, Vol. 407, Astrophysics and Space Science Library, ed. A. Lazarian, E. M. de Gouveia Dal Pino, \& C. Melioli (Berlin: Springer), 163

Beresnyak, A., Lazarian, A., \& Cho, J. 2005, ApJ, 624, L93

Biskamp, D. 2003, Magnetohydrodynamic Turbulence, ed. Biskamp, D. (Cambridge: Cambridge University Press)

Brandenburg, A., \& Lazarian, A. 2013, Space Sci. Rev., 178, 163

Chepurnov, A., \& Lazarian, A. 2010, ApJ, 710, 853

Chepurnov, A., Lazarian, A., Stanimirović, S., Heiles, C., \& Peek, J. E. G. 2010, ApJ, 714, 1398

Cho, J., \& Lazarian, A. 2003, MNRAS, 345, 325

Cho, J., Lazarian, A., \& Vishniac, E. T. 2002, ApJ, 564, 291

Cho, J., \& Vishniac, E. T. 2000, ApJ, 539, 273

Cho, J., Vishniac, E. T., Beresnyak, A., Lazarian, A., \& Ryu, D. 2009, ApJ, 693, 1449

Clegg, A. W., Cordes, J. M., Simonetti, J. M., \& Kulkarni, S. R. 1992, ApJ, 386,143

Eyink, G., et al. 2013, Nature, 497, 466

Falceta-Gonçalves, D., Kowal, G., Falgarone, E., \& Chian, A. C.-L. 2014 Nonlinear Processes in Geophysics, 21, 587

Goldreich, P., \& Sridhar, S. 1995, ApJ, 438, 763

Goodman, J., \& Narayan, R. 1985, MNRAS, 214, 519

Han, J. 2009, in IAU Symposium, Vol. 259, Cosmic Magnetic Fields: From Planets, to Stars and Galaxies, ed. K. G. Strassmeier, A. G. Kosovichev, \& J. E. Beckman, 455-466

Han, J. L., Ferriere, K., \& Manchester, R. N. 2004, ApJ, 610, 820

Haverkorn, M., Brown, J. C., Gaensler, B. M., \& McClure-Griffiths, N. M. 2008, ApJ, 680, 362

Haverkorn, M., Gaensler, B. M., McClure-Griffiths, N. M., Dickey, J. M., \& Green, A. J. 2004, ApJ, 609, 776

Haverkorn, M., Katgert, P., \& de Bruyn, A. G. 2003, A\&A, 403, 1045

Kóta, J., \& Jokipii, J. R. 2000, ApJ, 531, 1067
Kowal, G., Lazarian, A., \& Beresnyak, A. 2007, ApJ, 658, 423

Kritsuk, A. G., Norman, M. L., Padoan, P., \& Wagner, R. 2007, ApJ, 665, 416

Lazarian, A. 2005, in American Institute of Physics Conference Series, Vol. 784, Magnetic Fields in the Universe: From Laboratory and Stars to Primordial Structures., ed. E. M. de Gouveia dal Pino, G. Lugones, \& A. Lazarian, 42-53

Lazarian, A. 2009, Space Science Reviews, 143, 357

—. 2011, ArXiv e-prints:1112.3344

Lazarian, A., Esquivel, A., \& Crutcher, R. 2012, ApJ, 757, 154

Lazarian, A., \& Pogosyan, D. 2004, ApJ, 616, 943

-. 2006, ApJ, 652, 1348

-. 2012, ApJ, 747, 5

-. 2016, ApJ, 818, 178

Lazarian, A., \& Vishniac, E. T. 1999, ApJ, 517, 700

Maron, J., \& Goldreich, P. 2001, ApJ, 554, 1175

McKee, C. F. 1999, in The Origin of Stars and Planetary Systems, ed. C. J. Lada \& N. D. Kylafis (Dordrecht: Kluwer), 29

McKee, C. F., \& Ostriker, J. P. 1977, ApJ, 218, 148

Minter, A. H., \& Spangler, S. R. 1996, ApJ, 458, 194

Oppermann, N., et al. 2012, A\&A, 542, A93

Roy, S., Pramesh Rao, A., \& Subrahmanyan, R. 2008, A\&A, 478, 435

Simonetti, J. H., \& Cordes, J. M. 1986, ApJ, 310, 160

Simonetti, J. H., Cordes, J. M., \& Spangler, S. R. 1984, ApJ, 284, 126

Spangler, S. R. 1991, ApJ, 376, 540

Spangler, S. R., \& Gwinn, C. R. 1990, ApJ, 353, L29

Stil, J. 2009, in Panoramic Radio Astronomy: Wide-field 1-2 GHz Research on Galaxy Evolution, 45

Sun, X. H., \& Han, J. L. 2004, in The Magnetized Interstellar Medium, ed.

B. Uyaniker, W. Reich, \& R. Wielebinski, 25-30

Vestuto, J. G., Ostriker, E. C., \& Stone, J. M. 2003, ApJ, 590, 858

Xu, S., Yan, H., \& Lazarian, A. 2015, ArXiv e-prints

Zrake, J. 2014, ApJ, 794, L26 